\title{
Les médicaments de l'épilepsie vers 1800 *
}

Par F. Ledermann

A history of epilepsy cannot be written in the manner in which, for instance, a history of tuberculosis might be approached.

O. Temkin

Morbus caducus, morbus sacer, morbus deificans, morbus hereditarius, morbus major..., ${ }^{1}$ avec les nombreux éponymes de l'épilepsie, la sémantique indique que cette maladie n'est pas comme les autres, qu'elle occupe dans l'esprit du peuple, mais aussi sous le regard des médecins, une place à part. Avec ses signes à la fois mentaux et physiques - la chute, la salivation, les convulsions - l'épilepsie plus encore que d'autres maladies, oscilla longtemps entre le rationnel et le surnaturel, avec des composantes magiques, religieuses, cosmiques ou démoniaques ${ }^{2}$; elle balancera plus tard entre la psychiatrie et la neurologie ${ }^{3}$.

Reflet de ces orientations, l'usage de médicaments contre le mal caduc releva fréquemment d'une fantaisie débridée dans la recherche du plus étrange ou du plus repoussant, mais parfois aussi les drogues seront administrées sans originalité en fonction des grandes théories de la pathologie $^{4}$. Si l'histoire de la pharmacie, à travers ses multiples facettes, possède le médicament comme centre de gravité ${ }^{5}$, les drogues antiépileptiques servent à merveille de modèles pour une étude conceptuelle de l'histoire du médicament ${ }^{6}$. Pour évoquer, par exemple, la discordance, la distance, entre théorie médicale et emploi de produits pharmaceutiques ${ }^{7}$, pour souligner le rôle de quelques grandes questions relevant de la médecine (étiologie, hérédité, curabilité...) sur la thérapeutique ${ }^{8}$, pour examiner la place du spécifique, du produit doté d'une activité causale et en général de la spécificité de l'action des médicaments ${ }^{9}$, pour mesurer l'effet sur la matière médicale des modes, ou plus sérieusement des ruptures avec la tradition en raison ou non des progrès scientifiques et techniques, ou encore pour faire ressortir les différences entre la médecine officielle et les moyens thérapeutiques populaires.

* Texte d'une conférence donnée le 5 février 1986 à l'Institut d'histoire de la pharmacie de l'Université de Marburg (Pr R.Schmitz). 
Enfin la période étudiée qui va de 1770 à 1850 environ, ère de révision des idées en thérapeutique, d'un refus de la pharmacie, permet d'établir de quelle manière le nihilisme thérapeutique a atteint une maladie pour laquelle on avait proposé un nombre très grand de médicaments, et parmi les plus absurdes ${ }^{10}$.

\section{L'épilepsie et son traitement jusque vers 1750}

En 1851, le médecin anglais Locock introduit les bromures dans la thérapeutique de l'épilepsie. Cinquante ans plus tard, en 1912, Alfred Hauptmann administre du phénobarbital à des épileptiques, ouvrant par là une des indications majeures des barbituriques ${ }^{11}$. Une kyrielle de produits suivront tout au long du $20^{\mathrm{e}}$ siècle ${ }^{12}$. D'autre part la clinique de l'épilepsie connaîtra un grand bond en avant dans la seconde moitié du $19^{\mathrm{e}}$ avec les travaux de Jackson notamment ${ }^{13}$. Ces connaissances nouvelles sonneront le glas des conceptions hypothétiques et des moyens thérapeutiques souvent irrationnels, en tout cas hasardeux, qui prévalaient depuis des millénaires. Elles marquent un tournant dans l'histoire de cette maladie, toujours considérée jusque-là comme un peu à part. L'épilepsie devient alors un mal comme un autre, se normalise.

Certains textes assyro-babyloniens attribuent l'origine de l'épilepsie au dieu de la lune Sin. Aussi en Mésopotamie la thérapeutique consistait surtout en tentatives de purification, en incantations et en exorcismes; il s'agissait avant tout d'éviter toute contamination avec les esprits, causes de la maladie ${ }^{14}$. Ce concept de possession - qui n'est pas propre à l'épilepsie - se poursuivra au moins jusqu'au $5^{\mathrm{e}}$ siècle avant J.C., induisant des coutumes thérapeutiques particulières: le sang par exemple, et souvent le sang humain, usage dont l'origine doit être cherchée dans les sacrifices rituels ${ }^{15}$. Le sang des condamnés ou celui des gladiateurs morts dans l'arène était particulièrement prisés. Mais encore les os humains, le gui, le corail, la pivoine, la racine de strychnos ${ }^{16}$, préparés ou cueillis selon des rites précis, parfois portés en amulettes, ne sont que quelques exemples d'une longue liste de substances utilisées contre l'épilepsie. A cela s'ajoutaient les traitements de type religieux, en particulier par incubation dans les temples dédiés à Asclépios.

Visions magiques et surnaturelles combattues au $5^{\mathrm{e}}$ siècle a.C. par un traité de la collection hippocratique, le «De morbo sacro». Pour l'auteur de cet ouvrage, la maladie dite sacrée n'a rien de plus divin que les autres 
maladies; l'épilepsie a son origine dans le cerveau et survient quand les ventricules cérébraux sont envahis par le phlegme, ce qui empêche l'air de circuler ${ }^{17}$. Le rôle de l'hérédité, comme celui de la constitution de l'individu, sont reconnus ${ }^{18}$. A cette conception nouvelle de la maladie correspond un traitement qui rejette les pratiques magiques et religieuses et qui sans beaucoup d'illusion sur les possibilités réelles de la guérison - l'épilepsie est souvent reconnue comme incurable ${ }^{19}$ - préconise le régime, l'usage de certaines drogues, insiste sur la prévention. Les marches, les exercices, mais aussi les diurétiques, les purges et les émetiques - l'ellébore, par exemple sont appliqués par les tenants de la tradition hippocratique qui refusent donc au médicament un rôle particulier dans le traitement de l'épilepsie, les produits recommandés correspondant à la théorie émise sur la maladie: ainsi les drogues avec une action chauffante et desséchante, le castoréum ou la thériaque notamment, sont appliquées en priorité ${ }^{20}$.

Mais les conceptions magiques ou superstitieuses ne sont pas rejetées par tous et les deux visions de la maladie existent parallèlement. Certains médecins alexandrins, Sérapion par exemple, recommandent le poil de chameau, les selles de crocodiles, les testicules, le cœur ou le sang de divers animaux, ce genre de drogues figurant de manière abondante dans la littérature médicale de l'Antiquité. Il est impossible, souvent, de séparer de manière stricte les deux attitudes: les tenants de la pathologie humorale et d'une thérapeutique rationnelle admettant un effet thérapeutique - une action desséchante - à la racine de pivoine prescrite en amulette par les tenants d'une vision magique de l'épilepsie ${ }^{21}$.

Galien, par un effort de systématisation, tente une synthèse entre les différents courants ${ }^{22}$. Il établit un compromis entre une explication humorale et une explication mécanique, ou nerveuse, proche de la pathologie solidiste qui avait déjà été exprimée par certaines sectes d'Alexandrie. Inaugurant une classification qui restera valable longtemps après lui, il distingue une épilepsie due à une lésion idiopathique du cerveau, une épilepsie due à une modification du cerveau causée par le cœur et une épilepsie due à une modification du cerveau due à des troubles d'une autre partie du corps. Le fond du traitement consistait pourtant comme chez Hippocrate en préceptes diététiques et en purgatifs ${ }^{23}$, nourritures et boissons légères, exercices, miel, vinaigre, saignées, jus de scille. Il s'agit de rendre les humeurs qui se sont emparées du cerveau, moins lourdes, moins épaisses. En somme, à travers les diverses théories proposées, la médecine antique tente de donner une explication physiologique et anatomique de 
l'épilepsie. Sans pourtant obtenir des résultats thérapeutiques convainquants, ce qui entraîne la tentation constante d'user de produits bizarres; pour preuve la liste des médicaments contre l'épilepsie cités dans la Materia medica de Dioscoride ${ }^{24}$.

Au Moyen Age, avec le développement du christianisme, la notion de possession, par la lune, par d'autres astres mais aussi par le diable, se développe à nouveau: l'épileptique devient la victime d'une force surnaturelle qui a envahi son corps, la convulsion représentant les tourments endurés par le malade. L'épilepsie était généralement considérée comme contagieuse ${ }^{25}$. Chez Hildegard de Bingen et généralement au Moyen Age, le diable n'agit pas directement sur l'organisme mais seulement en cas de désequilibre humoral qui affecte le cerveau, lors de mauvais comportement moral, notamment; on trouve donc encore chez Hildegard une synthèse entre possession, morale et médecine ${ }^{26}$. Et nombreux sont les auteurs du Moyen Age, par exemple Arnauld de Villeneuve, qui cherchent une explication rationnelle à la matière médicale pléthorique et incongrue ${ }^{27}$. A ces idées, assez proches finalement de celles de l'Antiquité, s'ajoutera au Moyen Age le culte des saints et des reliques, en particulier le rôle de Saint-Valentin, avec ses pélerinages; ces pratiques religieuses se mêlant à l'administration de jus de pivoine, ou de gui, ou de houx: encore une synthèse ${ }^{28}$ !

La période des $16^{\mathrm{e}}$ et $17^{\mathrm{e}}$ siècles, est un temps où l'épilepsie est l'objet d'une attention accrue de la part des médecins; les observations, les études de cas, se multiplient et permettent de mieux connaître le développement et les symptômes de la maladie ${ }^{29}$. Toutefois, le traitement de l'épilepsie bénéficiera peu de cette amélioration de la clinique, restera inadéquat et surtout, l'étiologie de l'épilepsie reste mystérieuse, même si de 1500 à 1750 , les théories abondent pour tenter d'expliquer les causes du mal caduc. En fait, l'épilepsie n'échappe à aucune des modes, à aucune des grandes théories globalisantes de la médecine et de la thérapeutique; elle offre ainsi une figure paradigmatique pour une histoire des maladies ${ }^{30}$ ! Le rôle des forces démoniaques, même si elles n'agissent que par l'intermédiaire de causes naturelles est peu mise en doute. Et les drogues dont l'emploi est fondé sur des bases superstitieuses continuent à être administrées, même par des médecins considérés comme rationnels, comme Fernel, ou Weyer ${ }^{31}$.

Paracelse penche vers une conception cosmique de l'épilepsie. Dans le dualisme macrocosme-microcosme, le mal caduc est apparenté à un orage. Et son traitement constitue à mettre le malade à l'abri de l'influence des astres, grâce à des drogues, en agissant sur les conjonctions astrologiques. En 
plus des médicaments spagyriques habituels, de l'or et du corail, Paracelse recommande particulièrement trois produits: le gui, le sang d'un homme décapité, des parties de crâne humain, parant ces drogues, qui ne sont pas neuves, d'une efficacité contre les forces cosmiques ${ }^{32}$. Au $17^{\mathrm{e}}$ siècle encore, les conceptions de l'épilepsie s'inscrivent dans les théories explicatives de l'époque: pour Van Helmont, l'épilepsie se situe dans le centre de vie placé dans l'estomac et se libère par des émotions fortes, pour Borelli, les esprits animaux tordent les nerfs du cerveau, il en résulte une explosion des muscles. A ces tenants de la iatrophysique s'opposent les iatrochimistes, qui tels Sylvius ou Willis voient dans la cause de l'épilepsie un esprit volatil acide qui perturbe le fonctionnement des mouvements et des sens; le traitement se composant de médicaments basiques ${ }^{33}$. Toutes ces explications se concilient sans freins l'emploi des spécifiques, des médicaments bizarres et repoussants rencontrés tout au long de l'histoire de l'épilepsie.

\section{La situation à l'aube des Lumières: S. A.M.Tissot}

Les Lumières apportent un rejet du rôle des forces occultes et supranaturelles, des influences démoniaques, un refus aussi des grandes théories globalisantes. Mais le matérialisme médical et thérapeutique en train de naître s'appuie sur un renouveau des conceptions culturelles et philosophiques, sur un changement général d'attitude devant la nature plutôt que sur un progrès des sciences médicales.

Quel est l'effet des Lumières, vers 1770, sur le mal caduc et son traitement, le «Traité de l'épilepsie» du médecin suisse Samuel Tissot permet de le circonscrire. Disciple de Haller, connu pour son action et ses écrits en faveur de la santé publique ${ }^{34}$, Tissot publie à Lausanne en 1770 un ouvrage qui brise avec les conceptions anciennes sur l'épilepsie et construit les bases nouvelles de la clinique du mal caduc et de sa thérapeutique: le «Traité de l'épilepsie». ${ }^{35}$ Rejetant donc tout rôle des forces occultes, Tissot admet deux causes à l'épilepsie. La première, prédisposante, se trouve liée à une sensibilité particulière, à une disposition du cerveau à entrer en contraction ${ }^{36}$. Car Tissot, fidèle à l'influence de Haller, voit dans la compression et l'irritation des nerfs la cause première du mal ${ }^{37}$. L'autre cause, appelée déterminante, est «celle dont l'action met en jeu la première et qui se divise en sympathique et en idiopathique», ${ }^{38}$ l'auteur rappelant la distinction établie par Galien. 
L'épilepsie idiopathique est provoquée par des coups sur la tête, un vice de la conformité du crâne, mais aussi par une acreté des humeurs, une augmentation de la quantité du sang dans la tête, et surtout par des passions fortes ${ }^{39}$ : frayeurs, chagrins. Plus prosäquement, l'épilepsie sympathique est amenée par des troubles de l'intestin ou de l'estomac, par des vers intestinaux, par la grossesse, par les excès sexuels ou par les remèdes violents. Mais ces distinctions perdent chez Tissot le caractère dogmatique qu'elles avaient jusqu'aux Lumières car en s'appuyant sur une riche casuistique il nuance ensuite ses affirmations et procède à une individualisation de ses observations sur la maladie, traite l'épileptique plutôt que l'épilepsie.

Ce sens de la nuance qui confine souvent, fait nouveau, à un aveu d'ignorance domine dans les moyens thérapeutiques préconisés par Tissot; pour lui, soigner l'épilepsie, c'est «guérir toutes les causes occasionnelles, prévenir les accidentelles, changer la disposition épileptique du cerveau», ce qui «est toujours délicat... souvent difficile, quelquefois impossible» ${ }^{40}$. Le traitement doit être différencié selon les diverses formes d'épilepsie et selon la localisation de la cause ${ }^{41}$. Tissot, donc, s'attaquera à la cause prédisposante (à l'irritation, à la sensibilité des nerfs) par la trépanation, par la saignée, par des purgatifs, par des rafraîchissants, mais aussi par le régime, tout cela avec un seul but: éviter une quantité trop forte d'humeurs qui montent au cerveau ${ }^{42}$. En fait Tissot, sans se faire beaucoup d'illusions sur la possibilité d'un traitement de fond de l'épilepsie, dans l'ignorance qu'il est de la vraie cause de la maladie, prône plutôt un traitement d'apport, à base de vie saine, de mesures prophylactiques... et de quelques médicaments divers, purgatifs surtout. Il revient ainsi aux usages hippocratiques ${ }^{43}$, mais admet tout de même certains moyens d'agir: l'épilepsie pour lui n'est pas vraiment incurable!

La situation change pour l'épilepsie dite sympathique où Tissot peut agir sur des causes déterminantes somatiques. Le mal dont la cause est dans l'estomac est traitée par des vomitifs, ipécacuanha, kermès minéral..., quand elle est dans l'intestin, Tissot purge, sels neutres, rhubarbe, senné, jalap, casse, tamarins, poudre cornachine, si ce sont des vers qui produisent la crise, le semencontra, le kina ou le mercure la guérira, les excès vénériens sont combattus pas les toniques, fer, kina, mais aussi par la valériane ${ }^{44}$. En somme, Tissot ne refuse pas l'emploi des médicaments, ne pratique pas le nihilisme thérapeutique, mais veut porter une attention aux divers signes cliniques de la maladie pour la traiter. Et là se situe la rupture avec la tradition, le fait nouveau: non pas dans le choix des médicaments - ce sont les 
mêmes drogues que dans le passé - mais par la justification d'engager un traitement, par la réflexion sur l'utilité d'administrer des drogues.

C'est donc plus la prise en compte intellectuelle du médicament que son emploi qui est modifié; cela amène un autre regard sur la notion de spécifique, que Tissot refuse, bien qu'avec regret, semble-t-il: «de tous les remèdes, il n'y en a aucun qui mérite véritablement le nom de spécifique antiépileptique, parce qu'il n'y en a aucun qui guérisse certainement et constamment la disposition épileptique du cerveau, ni même aussi constamment que le kina guérit les fièvres... ou le mercure les maux vénériens». ${ }^{45}$

Ce refus du médicament étiologique s'appuie sur une remarque théorique, «en raison de la diversité des causes», ${ }^{46}$ mais aussi sur la clinique. Tissot, se fondant sur la littérature médicale des $17^{\mathrm{e}}$ et $18^{\mathrm{e}}$ siècles ainsi que sur ses propres observations, discute du mode d'action des nombreux produits réputés dans l'épilepsie, rejette ce qu'il appelle les «spécifiques inutiles», en particulier les médicaments dégoûtants et étranges, et ce qu'il nomme les «spécifiques dangereux», la teinture de cuivre, la graine de jusquiame, l'ellébore, le sang humain..., ne retenant qu'un nombre restreints de produits qu'il dit efficaces, mais pas spécifiques: la valériane surtout, qu'il appelle un antispasmodique, fidèle à l'idée de contraction des nerfs, mais aussi la feuille d'oranger, le musc, le gui, le kina, le camphre ${ }^{47}$. Pour ces drogues, Tissot tient compte des aspects pharmaceutiques; il met en garde contre la falsification des drogues (la racine de renoncule avec la valériane), il porte attention à la qualité des produits (la valériane ne doit pas être souillée par l'urine de chat), il indique parfois la dose des médicaments, préfère une forme galénique à une autre $^{48}$.

Mais Tissot use du paradoxe en matière de médicaments car pour l'épilepsie qui vient des organes, là où il croit utile l'usage des drogues, il ne fait qu'indiquer des classes thérapeutiques, sans discuter de l'action des médicaments pris individuellement: «Les émetiques entremêlés avec des purgatifs et dans l'entre-deux des huileux réussissent très bien». ${ }^{49} \mathrm{Et}$ pour la sensibilité du cerveau, quand Tissot doute du succès d'un traitement, il analyse longtemps l'effet des médicaments conseillés avant lui. Pourquoi cette contradiction? Peut-être parce que Tissot ne s'intéresse pas tant au traitement de l'épilepsie, qu'il sait peu accessible à des moyens médicamenteux, mais parce qu'il veut avant tout montrer son opposition aux doctrines anciennes, affirmer ce que sa pensée a de neuf.

Tissot, en rompant avec les conceptions anciennes sur les causes irrationnelles et sur les spécifiques, en s'exprimant sur la localisation de la maladie, 
en distinguant l'hystérie du mal caduc et en adoptant une attitude prudente et nuancée sur son traitement, annonce quelles seront les vues sur l'épilepsie jusque vers 1830. La question de l'hérédité de l'épilepsie, se fondant depuis 1820 environ, sur des études statistiques ${ }^{50}$, celle des signes cliniques de la maladie et du rôle du cerveau, la distinction entre épilepsie idiopathique et épilepsie sympathique, celles du rôle des excès sexuels, de l'onanisme, mais aussi des passions, des frayeurs ou encore de la grossesse, des écoulements sanguins ${ }^{51}$ continuent à être posées. Mais l'effort central autour de 1800 porte sur l'observation clinique, avec la recherche d'une lésion, et sur les distinctions nosographiques; sous l'influence des Français surtout, les concepts de grand mal, de petit mal, d'aura, sont peu à peu précisés et ce n'est plus surtout la crise qui intéresse les médecins. L'épilepsie est mieux distinguée des autres phénomènes convulsifs, et, proche encore de l'hystérie au milieu du $18^{\mathrm{e}}$ siècle avec Boerhaave, van Swieten ou Pomme, elle en est peu à peu séparée ensuite, chez Tissot déjà puis plus encore avec les représentants majeurs de la psychiatrie française, Pinel et Esquirol, notamment. Mais des liens se maintiennent entre le mal caduc et les maladies mentales, les épileptiques étant généralement internés dans les asiles de fous, les médecins étaient attentifs à leurs troubles psychiques ${ }^{52}$.

\section{La thérapeutique vers 1800}

La plupart des auteurs qui traitent du mal caduc vers 1800 admettent une forme idiopathique, qu'ils jugent généralement incurable, et une forme sympathique dont ils traitent les symptômes. Certains, qu'on peut voir comme les représentants de la tradition, comme Baumes qui publie en 1789 un ouvrage appelé «Des convulsions dans l'enfance», ${ }^{53}$ mais surtout le baron Portal qui fait paraître en 1827 ses «Observations sur la nature et le traitement de l'épilepsie», ${ }^{54}$ jugent l'épilepsie souvent incurable, mais surtout attachent une très grande importance aux causes et surtout aux signes somatiques de la maladie. Par ce biais, ils admettent un traitement médicamenteux, discutent de l'effet des drogues en fonction des signes cliniques et mettent en jeu une matière médicale étendue, mais traditionnelle, peu marquée par le progrès; Portal, par exemple, ne recuse pas a priori le concept de spécifique puisqu'il écrit: «sur quelques remèdes long-temps considérés comme spécifiques de l'épilepsie, quoiqu'ils ne le soient que dans des cas bien déterminés». ${ }^{55}$ Seules les drogues repoussantes sont rejetées. 
Cette attitude, dans laquelle on ne dénote aucun scepticisme à l'égard de l'usage de produits pharmaceutiques et qui tranche ainsi avec les idées du temps en thérapeutique - en psychiatrie surtout - est partagée par de nombreux autres médecins qui entre 1800 et 1830 écrivent sur l'épilepsie et son traitement. Les mêmes tendances apparaissent dans les ouvrages de médecine générale et de thérapeutique ainsi que dans les encyclopédies, les distinctions se faisant plus sur le choix des produits employés et sur le mode d'administration que sur les conceptions générales à l'égard des médicaments, peu empreintes d'aspects idéologiques. Bayle, par exemple, dans sa «Bibliothèque de thérapeutique», ${ }^{56}$ parue en 1828 , ne récuse ni les formes somatiques de l'épilepsie, ni les médications symptomatiques, il adopte seulement, attitude progressiste, des produits nouveaux comme la strychnine ou l'hydrocyanate de fer et donne de l'importance aux dosages et aux formes d'administration ${ }^{57}$.

Plus moderne, mieux adaptée à l'esprit de l'époque est l'attitude des représentants de la psychiatrie française, dominante alors, celle de Pinel, d'Esquirol et de leurs élèves ${ }^{58}$, qui dans le domaine des maladies mentales donnent la priorité aux causes morales, aux passions, et adoptent une méthode de soins, le traitement moral, héritée du sensualisme, qui privilégie les impressions sur le patient plutôt que les méthodes fortes et les drogues puissantes; ces conceptions sonnent le glas du «baroque thérapeutique» et entraînent une forme de scepticisme envers les drogues.

Mais l'attitude de prudence, sinon de refus, à l'égard des médicaments, la volonté de les soumettre à un examen critique avant de les employer, très prononcée pour l'ensemble des maladies mentales, est fortement atténuée, quand il s'agit de traiter l'épilepsie. Certes, le mal caduc est souvent vu comme incurable; ainsi Maisonneuve, un élève de Pinel, écrit que «sa résistance est presque insurmontable aux moyens curatifs», ${ }^{59}$ et Esquirol affirme que «l'épilepsie est désespérante par son incurabilité», ${ }^{60}$ mais tant Pinel, que Maisonneuve, ou encore Esquirol adoptent une attitude plus floue, moins nette, moins rigide de mise à l'épreuve des moyens thérapeutiques quand ils traitent de l'épilepsie plutôt que de la folie. On sent chez les représentants de cette école une inquiète incertitude: les médicaments leur paraissent ne pas servir à grand chose pour soigner - et surtout guérir - le mal caduc. Pour Esquirol, «c'est essentiellement aux secours de l'hygiène qu'il faut recourir», ${ }^{61}$ mais devant la gravité de la maladie, la puissance redoutable de ses symptômes, ont-ils le droit de ne rien faire? Alors que pour les maladies mentales, le traitement moral se substitue aux drogues en fonction 
d'une théorie - presque une idéologie - nouvelle et puissante, l'épilepsie échappe en partie à cette règle. Esquirol, plus loin: «Le quinquina, la valériane..., les analeptiques sont convenables». ${ }^{62}$

Ainsi, ils usent des remèdes, mais sans leur donner grande importance, sans enthousiasme, et surtout sans prôner tel produit plutôt que tel autre; Pinel, par exemple, écrit: «Je leur ai administré des bols de quinquina et de camphre... Les résultats indiquent que les remèdes doivent varier suivant les cas, et je me bornerai ici à remarquer que les effets... furent nuls pour trois de ces épileptiques, furent très-marqués pour les trois autres». ${ }^{63}$ Et Maisonneuve, rapporte: «il prit inutilement une grande quantité de valériane», ${ }^{64} \mathrm{et}$ s'étonne plus loin d'une guérison définitive obtenue avec de l'opium ${ }^{65}$. Reflet de cette indifférence sceptique à l'égard des drogues, le choix des médicaments reste traditionnel: valériane, feuille d'oranger, quinquina, camphre, asa-foetida, mais surtout, encore et toujours, les évacuants, coloquinte, jalap..., et les méthodes physiques, saignées, cautères, sétons ${ }^{66}$.

En somme, vers 1830, la médication de l'épilepsie, privée de ses aspects théatraux du passé, de sa dimension magique, de son pouvoir étiologique, réduit également par la lutte contre la polypharmacie, joue un rôle annexe, un peu disturbateur car finalement personne ne s'en passe; on pourrait parler d'un empirisme détaché, indifférent.

Après 1830

Plus tard, le développement de l'anatomie pathologique, concentrée sur la physiologie plutôt que sur la recherche du site anatomique provoque une rupture presque complète entre l'avance de la médecine et l'usage de médicaments contre l'épilepsie ${ }^{67}$. Au début du $19^{\mathrm{e}}$ siècle, les discussions sur la matière médicale comme celles sur l'emploi ou non de produits pharmaceutiques «collent» encore à la théorie; vers 1850, avec les travaux de Delasiauve, de Herpin, puis ceux de Morel et de Griesinger entre autres, l'esprit plus scientifique avec lequel s'exerce la recherche médicale - on pensera à Claude Bernard - n'induira pas de véritable réflexion sur les médicaments: le développement de la thérapeutique prendra une voie séparée de celui de la médecine, avec d'autres concepts de progrès.

On peut voir une image de cette inadéquation dans le concept de curabilité de l'épilepsie: alors que le Genevois Théodore Herpin, médecin de ville, la juge très souvent curable ${ }^{68}$, Delasiauve, médecin de l'asile d'aliénés 
de Bicêtre, écrit qu'elle «conduit presque infailliblement à l'incurabilité ${ }^{69}$. Mais tous deux discutent ensuite longuement de thérapeutique, et presque dans les mêmes termes. Car, après 1830 et surtout vers 1850, l'administration des médicaments du mal caduc répondra à deux éléments jusque là diffus et plutôt indépendants du système de pensée de la médecine. Le premier, avec Herpin notamment, c'est l'irruption de la statistique dans la manière de juger les produits pharmaceutiques ${ }^{70}$, phénomène issu de la médecine asilaire mais qui participe surtout d'une volonté de donner un visage scientifique au médicament, de privilégier l'empirisme au détriment des systèmes. Le second élément nouveau est directement lié au développement de la pharmacie: alors que la matière médicale, jusque vers 1830 , avait peu changé puisque le rapport mental, intellectuel au médicament seul s'était modifié, plus tard l'épilepsie est traitée avec des produits nouveaux, avec des alcalö̈des, strychnine et quinine notamment, et plus largement avec des sels métalliques, de zinc, de cuivre, d'argent...

Cette modernisation, cette adaptation de l'arsenal thérapeutique aux progrès de la pharmacie et de la chimie s'accompagne d'une importance plus grande donnée aux aspects strictement pharmaceutiques du médicament; les problèmes de dosages, le choix de la forme pharmaceutique adéquate ${ }^{71}$, la préoccupation d'éventuels effets secondaires néfastes font irruption dans les textes traitant de l'épilepsie. Ainsi Delasiauve écrit-il à propos du nitrate d'argent: «Sauf les cas exceptionnels, où les doses ont été portées à 10, 15 et même 30 centig., on n'a point franchi la limite de 5 centig. que nous avons nous-même respectée... On donne souvent le nitrate d'argent en pilules. Fouquier l'administre dans une tisane ou en juleps... la réflexion nous conduisant à préférer un véhicule liquide. N'a-t-on pas à... craindre... que le caustique... ne détermine des altérations plus ou moins graves». ${ }^{72}$ Et Herpin renchérit: «le remède donné sous le nom d'oxyde de zinc a dû être presque toujours l'oxyde préparé par combustion, ou fleurs de zinc; cependant il a pu être aussi la même substance obtenue par précipitation; car nous savons que, dans l'une de nos pharmacies, on donne sur prescription d'oxyde cette dernière préparation; mais cette pharmacie donne toutes garanties d'une pureté parfaite du médicament». ${ }^{73}$

Ce texte, qui n'est pas isolé ${ }^{74}$, témoigne de l'orientation nouvelle pharmaceutique et pharmacologique - du traitement de l'épilepsie. Ces améliorations amènent un retour à une prise en compte plus active, plus positive, du traitement médicamenteux; on discute alors de nouveau de l'action de multiples produits, ce qui s'accompagne d'une redécouverte de 
bon nombre de drogues traditionnelles ${ }^{75} \mathrm{et}$, fait curieux, d'un retour assez marqué du concept de spécifique. Herpin, ainsi, se fait l'ardent propagateur du zinc: «L'oxyde de zinc est, de tous les médicaments que nous avons employés, celui dont nous avons fait le plus fréquent usage.» ${ }^{76}$ Et Morel, pourtant encore partisan souvent des méthodes morales, recommande la belladonne et le sulfate de quinine ${ }^{77}$. Ces démarches et l'application d'un empirisme sans freins permet même un retour aux produits bizarres puisque Herpin va jusqu'à admettre que «la taupe grillée a guéri quelques épileptiques». ${ }^{78}$

Les nouvelles substances, les progrès de la pharmacie coïncident avec une utilisation à nouveau plus large des drogues, surtout avec une réintroduction d'un visage fabuleux, d'un caractère particulier donnés aux médicaments de l'épilepsie. Mais l'attitude des médecins envers les médicaments, qui va d'un emploi sans bornes - voire sans réflexion - à un rejet parfois immotivé n'est en rien fonction du progrès des sciences pharmaceutiques; c'est manifeste avec Tissot dont la pensée réflète les premiers feux des Lumières, et c'est toujours vrai autour de 1850. A cette époque, les vues théoriques sur le médicament dépendent du cadre intellectuel et culturel et non pas de l'environnement scientifique; l'épilepsie sert ici de modèle pour une assertion qui vaut pour l'ensemble de la thérapeutique.

\section{Zusammenfassung}

Die medikamentöse Behandlung der Epilepsie um 1800

Am Beispiel der Epilepsie wird die Haltung der Ärzte gegenüber der medikamentösen Behandlung zwischen 1770 und 1850 aufgezeichnet. Die Einstellung zur Arznei, die von einem manchmal unmotivierten Verwerfen bis zu einer maßlosen Verwendung schwankte, hängt nicht so sehr vom wissenschaftlichen, medizinischen und pharmazeutischen Umfeld, sondern vielmehr von den kulturellen und ideologischen Zeitanschauungen ab.

\section{Notes}

1 Sur ce point, comme sur beaucoup d'autres, cf. O.Temkin, The Falling Sickness, $2^{e}$ éd., Baltimore, 1971, p.7; et aussi L.Kanner, The Names of the Falling Sickness An Introduction to the Study of the Folklore and Cultural History of Epilepsy, Human Biol. 2, 1930, p.109-127, M.Cipriani, Contributo allo studio dei vecchi appellativi agiografici del mal caduco, Riv. Stor. Med. 10, 1966, p.94-117, M. Cipriani, «Mater puerorum» sinonimo di epilessia secondo vecchie concezioni etiologiche, Riv. Stor. Med. 14, 1970, p.172-179, 
A. Mechler, Zur Eponymik der Epilepsie, Med. Welt 151, 1963, p.535-538, K. Dieckhöfer, Die «Volksversammlungskrankheit» im alten Rom. Ein Beitrag zur Geschichte der Epilepsie, Schweiz. Arch. Neurol. Neurochir. Psychiatr. 40, 1972, p.317-329, E. W. Fungfeld, "Morbus sacer» Ein Überblick vom Altertum bis zur Gegenwart, Med. Welt 17, NF, 1966, p.258-266. Voir aussi E.Esquirol, Des maladies mentales considérées sous les rapports médical, hygiénique et médico-légal, Paris 1838, p.274, et L.J.F. Delasiauve, Traité de l'épilepsie, Paris 1854, p.21-23.

2 Ce balancement entre deux visions antagonistes du mal est orienté plutôt vers le magique là où le soignant ne constatait aucune lésion corporelle: chez Homère déjà, la pharmacie de guerre est plutôt empirique alors que la thérapeutique des désordres mentaux, par exemple, restera encore longtemps soumise aux bizarreries du surnaturel. Sur ce thème, ef. F.J. Kuhlen, Zur Geschichte der Schmerz-, Schlaf- und Betäubungsmittel in Mittelalter und früher Neuzeit, Stuttgart 1983, O. Temkin, op.cit. p.3, M. H.Charlton, Hippocrates and Asclepios, NY State J. Med. 75, 1975, p.117-119, et J. L.Pasternak, An Analysis of Social Perceptions of Epilepsy; Increasing Rationalization as Seen Through the Theories of Comte and Weber, Soc. Sci. Med. 15, 1981, p.223-229.

3 H. Beauchesne, La connaissance médicale de l'épilepsie pendant la première partie du $19^{e}$ siècle, Persp. Psychiatr. 16, 1978, p.37-42, et G. E. Berrios, Epilepsy and Insanity During the Early 19th Century, Arch. Neurol. 41, 1984, p. 978-981.

4 D. Janz, Leitbilder der Epilepsie bei Hippocrates und Paracelsus, Jahrb. Psychol. Psychiatr. 14, 1966, p.2: "Wie bei einem so augenfälligen Geschehen nicht anders zu erwarten, waren - und sind heute noch - die pathogenetischen Theorien immer bildhafte Vorstellungen von natürlichen oder übernatürlichen Vorgängen - Leitbilder sozusagen, in denen sich die jeweilige Ansicht vom Wesen der Krankheit widerspiegelt.»

5 R.Schmitz, Pharmaziegeschichte heute - Versuch einer Standortbestimmung, Apoth. J. 1983, p. 10-18.

6 F. Ledermann, La psychiatrie française et les médicaments: Pomme, Pinel, Esquirol, Morel, Rev. Hist. Pharm. XXIX, no 254, 1982, p. 189-206, et F. Ledermann, La thérapeutique médicamenteuse et la psychiatrie allemande du XIX $X^{e}$ siècle, concordances, oppositions, indifférence? Gesnerus 39, 1982, p.451-467.

7 J. Pigeaud, Les mains des dieux, quelques réflexions sur les problèmes du médicament dans l'Antiquité, Littérature, Médecine, Société, nº 4, 1982, p. 65.

8 I.D. Melville, The Medical Treatment of Epilepsy: A Historical Review, in F.Clifford et W. Bynum, Historical Aspects of the Neurosciences, New York 1982, p.127-136.

9 L. Belloni, Dall'elleboro alla Reserpina, Arch. Psi. Neurol. Psichiatr. 117, 1956, p. 45-133, et J.Starobinski, Histoire du traitement de la mélancolie des origines à nos jours, Bâle 1960.

10 E. Esquirol, op. cit. p. 317.

11 C. Locock, Contribution to discussion on paper by E.H.Sieveking, Lancet, $\mathrm{n}^{\circ} 1,1857$, p.528, et A.Hauptmann, Luminal bei Epilepsie, Münch. Med. Wschr. 59, 1912, p. 1907-1909. Voir aussi H. Schneble, «Antiepileptische Therapie 1877»-Die Behandlung der Epilepsie vor 100 Jahren, Nervenarzt 49, 1978, p. 76-81.

12 H.D.Pache, Historisches zur Epilepsie-Diagnostik und -Therapie, Münch. med. Wschr. 120, 1978, p.1723-1724, et D.B.Smith et al., Historical Perspective on the Choice of Antiepileptic Drugs for the Treatment of Seizures in Adults, Neurology 33, 1983 (3 Suppl.1), p.2-7. 
13 O.Temkin, op.cit.p.303, R. H. Wilkins et A.Brody, Jacksonian Epilepsy, Arch. Neurol. 22, 1970, p. 183 sq., et H. Heintel, Quellen zur Geschichte der Epilepsie, Stuttgart/Wien 1975, p. 87 sq. Voir aussi A.B.Brazier, The Search for the Neurological Mechanisms in Epilepsy: an Overview, Neurology 24, 1974, p.903-911, et D.P.Purpura, An Historical Study of Neurophysiological Concepts in Epilepsy, Epilepsia, 3 ${ }^{\text {e }}$ s. 1953, p.115-126.

14 O. Temkin, op. cit. p. 3-12; pour une comparaison avec les civilisations précolombiennes, cf. F. Sal y Rosas, Indicios de la epilepsia en el Peru antiguo, Rev. Neuropsiqu. 33, 1970, p.31-44, et D. Vasconcelos, La enfermedad sagrada. Aspectos historicos y situación actual, Gac. Med. Mex. 118, 1982, p. 35-37.

15 O.Temkin, op. cit. p. 12.

16 Ibid., p. 13.

17 Ibid., p. 4-5; voir aussi R. Joly, Hippocrate médecine grecque, Paris 1964, p. 88-108.

18 W. Leibbrand et A. Wettley, Der Wahnsinn, Geschichte der abendländischen Psychopathologie, Freiburg/München 1961, p.39, R.Bing, Bemerkungen zur Frühgeschichte der Epilepsie, Schweiz. Med. Wschr. 85, 1955, p. 97-102, et D. Janz, Leitbilder..., op. cit. p.5.

19 O. Temkin, op. cit. p.65, et R. Joly, op. cit. p. 102.

20 Pour Aretée, cf. O.Temkin, op. cit. p.68-69, et pour Celse, cf. E. Ackerknecht, Therapie von den Primitiven bis zum 20. Jahrhundert, Stuttgart 1970, p.30. Voir aussi E. Venkovski, Medizinisch-historischer Überblick über die epileptische Krankheit und über ältere Anschauungen zur Therapie der Epilepsie, Psychiatr. Neurol. Psychol. 24, 1972, p. 508-515.

21 O. Temkin, op. cit., p. 22-26.

22 Ibid., p. 60.

23 Ibid., p. 31 et passim, et K. Dieckhöfer, op. cit., ainsi que K. Dieckhöfer, Die Epilepsie im Zitat der römischen Schriftsteller Plautus, Seneca und Apuleius, Confin. Psychiatr. 65, 1972 , p. 212-219.

24 O.Temkin, op. cit. p. 79-80.

25 Cf. W. Gottwald, Die Epilepsie als Infektionskrankheit vornehmlich im Spiegel medizinhistorischen schlesischen Schrifttums des Mittelalters, Med. Welt 41, 1969, p. 2274-2277.

26 Cf. W.-D. MüllerJahncke, Astrologisch-magische Theorie und Praxis in der Heilkunde der frühen Neuzeit, Stuttgart 1985, p. 217: «Dies bedeutet, daß beispielsweise die Eigenschaft des Magneten, Eisen anzuziehen, oder diejenige der Pfingstrose, die Fallsucht zu heilen, nicht durch von den Gestirnen ausgehende Emanationen vermittelt wird, sondern nur durch die Wärme.»

27 O.Temkin, op. cit. p.104, et G.Roth, Epilepsia est morbus non possessio, Confin. Psychiatr. 13, 1970, p. 67-72.

28 Par exemple, cf. S. Bergmans, Un fragment peint du pélerinage des épileptiques à Moleenbeeck-Saint-Jean, æuvre perdue de Pierre Breughel l'Ancien, Rev. Belge Archéol. Hist. Art 41, 1972, p.41-57, H.Doose, Aus der Geschichte der Epilepsie, Münch. Med. Wschr. 107, 1965, p.180-191, R. Laulan, Les guérisons miraculeuses des écrouelles et de l'épilepsie par les rois d' Angleterre, Presse méd. 73, 1965, p.2707-2709.

29 O.Temkin, op. cit. p.182, et J.Bieder, Aperçu sur l'état des connaissances neuropsychiatriques au XVII e siècle d'après l'œuvre de Michel Ettmüller, Ann. méd. Psychol. 2, 1975, p. 783-791.

30 D. Janz, Leitbilder..., op. cit. p. 2. 
O. Temkin, op. cit. p. 138-145.

32 Ibid.p.176, D.J Janz, Leitbilder..., op. cit. p. 10-14, et H. Heintel, op. cit. p. 37-43.

33 O. Temkin, op. cit. p. 182-218, H. Heintel, op. cit. p.52, K. Dieckenhöfer, Die Epilepsie an der Wende zum 18.Jahrhundert, Schweiz. Archiv Neurol. Neurochir. Psychiatr. 111, 1972, p.89-97, R.F. Kraus, Concepts of Epilepsy in Seventeenth-Century England, Trans. Stud. Coll. Physic. Philad. 40, 1974, p.244-247, et T. Mildner, Die Behandlung der "Fallenden Sucht» im 18. Jahrhundert, Med. Klin. 61, 1966, p. 2914-2917.

34. Linoculation, par exemple. Cf. O. Temkin, op. cit. p. 227, K. Karbowski, Samuel Auguste Tissot et son "Traité de l'épilepsie» de 1770, Lausanne 1984, H. Bucher, Tissot und sein Traité des nerfs, Zurich 1958, et K. Karbowski, Das «Traité de l'épilepsie» von S. A. Tissot; alt, aber nicht veraltet, Praxis 73, 1984, p. 751.

35 S. A. M. Tissot, Traité de l'Epilepsie, Lausanne 1770.

36 Ibid.p.26.

37 Ibid.p. 25.

38 Ibid.p. 226.

39 Ibid. p. 154 et passim.

$40 \quad$ Ibid.p. 229.

41 Ibid. p. 224.

42 Ibid.p.267-271.

43 O. Temkin, op. cit. p. 240 ; ce qui annonce la grande époque de la psychiatrie française.

44 S.A.M.Tissot, op. cit. p. 246.

45 S.A.M.Tissot, op. cit. p.300-301, et P. Morel et C. Quétel, Les médecines de la folie, Paris 1985, p. 78.

46 S.A.M. Tissot, op. cit. p. 229.

47 Ibid. p. 300 sq. Tissot rejette de nombreux autres produits qu'il juge inefficaces, le castor, le fer, l'ase fétide, la rue etc. Sur les antispasmodiques, ef. P. Morel et C.Quétel, op. cit. p. 131-132.

48 S. A.M. Tissot, op. cit. p. 314 et passim.

49 Ibid. p. 233.

50 O. Temkin, op. cit. p. 260 sq.

51 Sur les castrations et les clidorectomies qui en ont résulté, cf. ibid. p.230, ainsi que P. Morel et C. Quétel, op. cit.

52 O.Temkin, op. cit. p.224-259, H.Beauchesne, La connaissance médicale de l'épilepsie pendant la première partie du $19^{e}$ siècle, Persp. Psychiatr. 16, 1978, p.37-42, et J.L.O'Leary et S.Goldring, Science and Epilepsy, Neurosciences Gains in Epilepsy Research, New York 1976, p. 15-34. G. E. Berrios, Epilepsy and Insanity During the Early 19th Century, Arch. Neurol. 41, 1984, p.978-981, et G. H.Glaser, Epilepsy, Hysteria and «Possession», J. Nerv. Ment. Dis. 166, 1978, p. 268-274.

53 M. Baumes, Des convulsions dans l'enfance, Paris 1789.

54 P. Portal, Observations sur la nature et le traitement de l'épilepsie, Paris 1827.

55 Ibid. p. V.

56 A.J.L.Bayle, Bibliothèque de Thérapeutique ou Recueil de mémoires originaux et des travaux anciens et modernes sur le traitement des maladies et l'emploi des médicaments, Paris 1828.

57 Ibid. p. 62: «...épilepsie probablement guérie par le phosphore». 
58 R. Semelaigne, Les pionniers de la psychiatrie française avant et après Pinel, Paris 1930, Y.Pelicier, Histoire de la psychiatrie, Paris, $2^{\mathrm{e}}$ éd., 1971, et H.Baruk, La psychiatrie française de Pinel à nos jours, Paris 1967.

59 J.G.F. Maisonneuve, Recherches et observations sur l'épilepsie, suivies d'un Tableau des Genres et des Espèces de cette Maladie, avec l'indication du traitement qui leur Convient, Paris 1803.

60 E. Esquirol, op. cit. p. 282.

$61 \quad$ Ibid. p. 318.

62 Ibid. p.324.

63 P. Pinel, Nosographie philosophique, ou la méthode de l'analyse appliquée à la médecine, Paris 1803, p.96. Il n'est pas question de l'épilepsie dans le Traité médico-philosophique sur l'aliénation mentale, Paris 1809.

64 J.G.F. Maisonneuve, op. cit. p. 25.

65 Ibid.p.77.

66 P. Pinel, Nosographie..., op. cit. p.96-97, E. Esquirol, op. cit. p.317sq., et J. G. F. Maisonneuve, op. cit. p. 66.

67 K. Doerner, Bürger und Irre, Frankfurt 1969, p. 140.

68 T. Herpin, Du pronostic et du traitement curatif de l'épilepsie, Paris 1852, p. 23.

69 L. J.F. Delasiauve, op. cit. p. 282.

70 T. Herpin, op. cit. p.576 et passim, G. E. Berrios, op. cit. p. 980, et D. Janz, Die Epilepsien, Stuttgart 1969, p. XIV.

71 Cf. par exemple B.A. Morel, Traité des maladies mentales, Paris 1860, p. 571.

72 L. J. F. Delasiauve, op. cit. p. 393.

73 T. Herpin, op. cit. p. 558.

74 B. A. Morel, op. cit. p. 753-755.

75 Cf. L. Kanner, Mistletoe, Magic and Medicine, Bull. Hist. Med. 7, 1930, p.929-933.

76 T. Herpin, op. cit. p.555. Sur l'emploi antérieur de l'oxyde de zinc, cf. P. Morel et C. Quétel, op. cit. p. 131.

77 B. A. Morel, op. cit. p. 753-755.

78 T. Herpin, op. cit. p.597. Cet effacement du nihilisme thérapeutique n'est donc pas lié directement à l'introduction des bromures, comme le prétend Ritter, mais la précède; les phénomènes idéologiques annonçant les changements scientifiques ou techniques. Cf. G. Ritter, Bemerkungen zur Sozialgeschichte der Epilepsie, Nervenarzt 46, 1975, p.513-578, ou encore G. Ritter, Epilepsie und soziales Vorurteil in historischer Sicht, Psychiatr. Neurol. med. Psychol. 25, 1973, p. 754-761. 


\section{Summary}

Taking epilepsy as an example, a record is made of the way of thinking doctors had of medical treatment between 1770 and 1850 . The attitude towards drugs which wavered at times between an unmotivated rejection and an abusive use is less due to scientific, medical and pharmaceutical environment than to the cultural and ideological mentality of the period.

$\mathrm{D}^{\mathrm{r}}$ François Ledermann

Pharmacien

Kirchbühlweg 21

CH-3007 Berne 